\title{
Efficient Sensor-Cloud Communication using Data Classification and Compression
}

\author{
Md. Tanvir Rahman \\ Department of Computer Science and Engineering, Daffodil International University, Dhaka, 1207, Bangladesh \\ E-mail: sajal.it.ju@gmail.com \\ Md. Sifat Ar Salan \\ Department of Statistics, Jahangirnagar University, Dhaka, 1342, Bangladesh \\ E-mail: sifat246@yahoo.com \\ Taslima Ferdaus Shuva \\ Department of Computer Science and Engineering, Daffodil International University, Dhaka, 1207, Bangladesh \\ E-mail: shuva.it.ju@gmail.com \\ Risala Tasin Khan \\ Institute of Information Technology, Jahangirnagar University, Dhaka, 1342, Bangladesh \\ E-mail: risala@juniv.edu
}

\begin{abstract}
Wireless Sensor Network, a group of specialized sensors with a communication infrastructure for monitoring and controlling conditions at diverse locations, is a recent technology which is getting popularity day by day. Besides, cloud computing is a type of high-performance computing that uses a network of remote servers which simultaneously provides the service to store, manage and process data rather than a local server or personal computer. An architecture called sensor-cloud is also providing good services by combining the capabilities from both ends. In order to provide such services, a large volume of sensor network data needs to be transported to cloud gateway with a high amount of bandwidth and time requirement. In this paper, we have proposed an efficient sensor-cloud communication approach that minimizes the enormous bandwidth and time requirement by using statistical classification based on machine learning as well as compression using deflate algorithm with a minimal loss of information. Experimental results describe the overall efficiency of the proposed method over the traditional and related research.
\end{abstract}

Index Terms-Wireless Sensor Network, Cloud Computing, Classification, Compression, Sensor-Cloud Communication.

\section{INTRODUCTION}

Cloud computing and wireless sensor network (WSN) are two of the recent technologies which have got huge popularity in the field of information and communication technology. Wireless storage system being a part of the wireless network has not been used in large-scale applications because of having many issues such as limited bandwidth, unreliable channel, heterogeneity and so on [1]. After integrating WSN with cloud environment these shortcomings are surpassed.

By introducing compression algorithms, the storage limitation of sensors is also overcome in sensor devices [2]. With these algorithms, the size of data and transmission energy is reduced [3]. But data compression will be significant if execution of algorithm does not require more energy than each transmission [4]. This problem is overcome by completely moving the compression process to the gateway.

The authors of [5] proposed a model where the communication between WSN and cloud is based on gateways. Data are collected at each sensor (deployed at various places) and directly sent to sensor gateway without any manipulation at sensor end. The collected data are passed through neural network and then compressed for reducing transmission. As the processing is done at sensor gateway, power consumption at sensor node is reduced.

For improving accuracy, the interval between each consecutive data collection must be kept minimal. As a result, the data size is becoming very large. Since the time interval is equal, after completing a cycle (e.g. a month or a year) there will be a lot of similar data because of seasonality. Again, data duplication occurs when the environment does not change its state rapidly.

In this research, we have observed the basic characteristics of sensor-cloud infrastructure, the appropriate communication medium and the network architecture of it. We have also identified the key challenges of sensor-cloud communication and targeted a problem of huge bandwidth consumption for real time sensor-cloud applications. From this context, we have proposed an efficient communication framework to bring the bandwidth issue under control and thus improving the 
efficiency of a sensor-cloud architecture. We have further implemented a demo application and made a comparison between the traditional and proposed framework as well as with related research.

The main contributions of the paper are as follows: firstly, to be the best of our knowledge, this paper is one of the first to systematically study the bandwidth requirement issue for sensor-cloud communication. Secondly, we have proposed how to reduce the bandwidth consumption with a minimal loss of information during the transaction between sensor network and cloud environment by incorporating the concept of machine learning approach with data classification and compression techniques. Thirdly, we have also considered the required time for our framework and then compared with the traditional approach. Finally, we have manually investigated the overall bandwidth consumption and time requirement with related works.

The remainder of the paper is organized as follows: in section II, an overview of sensor-cloud architecture is presented. In section III, a summary of similarity-based classification is discussed. The problem statement for the proposed work is explained in section IV. Section V presents the proposed framework along with an activity diagram. A detailed analysis and implementation of the framework is illustrated in section VI. Finally, in section VII, a detailed explanation of the simulation results is presented. The section VIII summarizes the proposed work with concluding statements.

\section{SENSOR-CLOUd INFRASTRUCTURE}

Sensor-Cloud infrastructure combines WSN and cloud computing in a way that can produce a powerful and ondemand performance access for real-time data processing and storage of sensor network data as well as the analysis of the processed information to reveal hidden sights. This combined infrastructure can be treated as an extension of cloud computing that can manage the physical sensors of WSN in order to meet the increasing demand for large scale wireless network applications [6].

\section{A. Wireless Sensor Network}

A wireless sensor network (WSN), sometimes called wireless sensor actuator network (WSAN), is defined as a collection of spatially distributed autonomous sensors. These sensors typically have low processing power and storage availability [7]. For any monitoring and controlling application, these tiny sensors can sense, measure and gather information from the environment and transmit the data to the user.

A WSN typically does not have any certain infrastructure. As a result, it can be categorized into two broad types such as structured and unstructured. For the unstructured WSN, the physical sensors are deployed in an ad-hoc manner whereas in structured network there must be a pre-plan for deploying whether all or some of the sensor nodes [8].

The application areas of wireless sensor networks are weather forecasting [8], military command and control [9,
10], natural disaster relief management [11], e-health [12, 13] and so on.

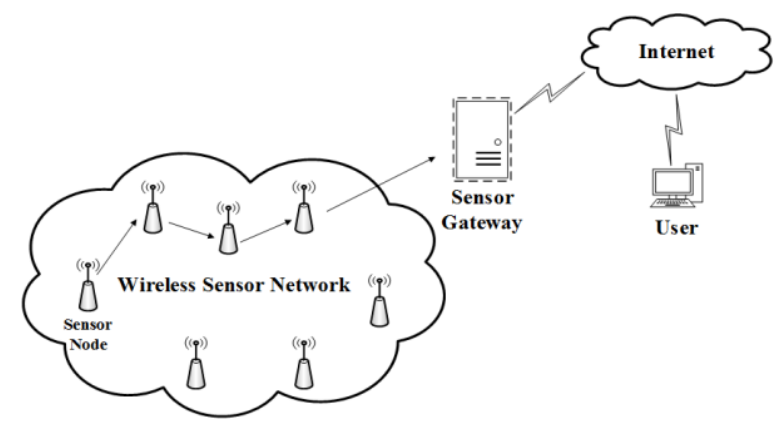

Fig.1. Wireless Sensor Network

\section{B. Cloud Computing}

Cloud computing, also on-demand computing, is a type of internet based computing that provides an on-demand service access through shared processing resources [14]. The term cloud in cloud computing is used as a metaphor for 'the internet'. As a result, all of the cloud services (e.g. software, platform, infrastructure etc.) are delivered to the user through the internet.

Cloud computing can be thought as a model of network computing where the servers can be in the form of virtual machines or physical machines in the cloud. To achieve coherence, it relies on the resources to be shared through different cloud services [15].

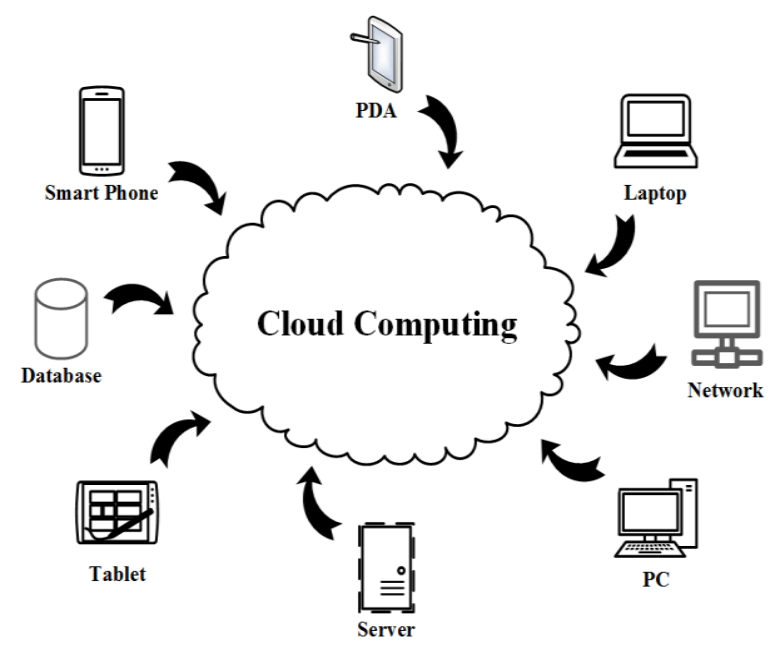

Fig.2. Cloud Computing

Cloud computing also provides great and convenient user experience because the end users don't need to think about the actual location of the servers. They can have the service by simply connecting to the server using a login panel [6].

\section{Integration of WSN and Cloud Computing}

In sensor-cloud infrastructure, the WSN is integrated with cloud environment in order to achieve convenient processing and storage. This approach allows the sensor network to accumulate and transmit all sensor data to cloud in a periodic time interval. 
The sensor-cloud infrastructure (i.e. the integrated infrastructure of WSN and cloud computing) is a unique sensor data storage, analyzing and monitoring platform that uses scalable cloud computing approach to providing excellent data analysis and visualization [16].

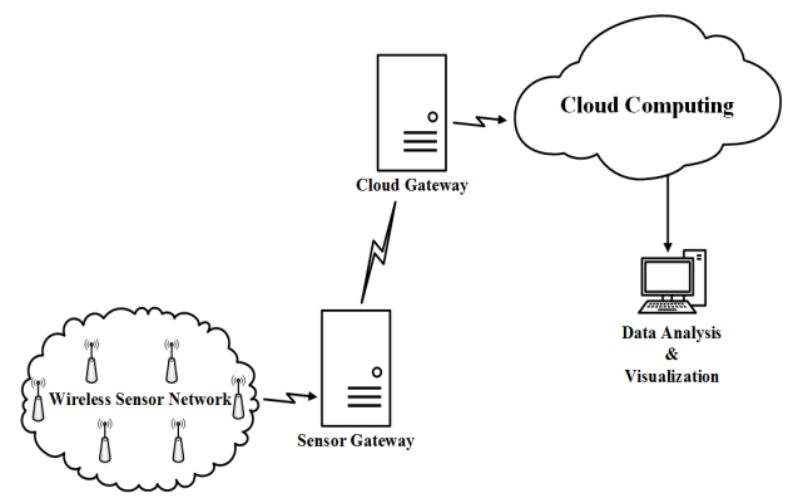

Fig.3. Sensor-Cloud infrastructure

In this approach, the limitations of WSN such as limited storage, processing, and power consumption are overcome. As cloud computing has massive storage and processing capability, it enables the sensor network to collect the huge amount of data by connecting it to the cloud through gateways. The sensor gateway collects the sensor network data and after compression, it sends the data to cloud gateway through the internet.

\section{SiMILARITY-BASED CLASSIFICATION AND COMPRESSION}

\section{A. Similarity and Distance}

The purpose of similarity and distance measure is to compare two sets of data (record, vector) and calculate a single value that represents their similarity. These measures are essential to understand the closeness property of two data sets. But choosing an appropriate similarity measure is also important for classification. There are several ways to measure the distance for a different pattern of data sets in classification and clustering. Some of them are as follows:

\section{1) Euclidean Distance:}

Euclidean distance [17] is a special case of Minkowski distance. It is the (straight line) distance between two points in two-dimensional space [18]. In Cartesian coordinates, if $X=\left(x_{1}, x_{2}, \ldots, x_{n}\right) \quad$ and $Y=\left(y_{1}, y_{2}, \ldots, y_{n}\right)$ are two points, then the distance $(d)$ from $X$ to $Y$ or from $Y$ to $X$ is,

$$
\begin{aligned}
d & =\sqrt{\left(x_{1}-y_{1}\right)^{2}+\left(x_{2}-y_{2}\right)^{2}+\cdots+\left(x_{n}-y_{n}\right)^{2}} \\
& =\sqrt{\sum_{i=1}^{n}\left(x_{i}-y_{i}\right)^{2}}
\end{aligned}
$$

\section{2) Cosine Similarity:}

Cosine similarity [19] is a kind of similarity measure between two objects that calculates the cosine of the angle between them. It evaluates the judgement of orientation rather magnitude (i.e. the cosine similarity of two vectors with the same orientation is 1 , if the angle between them is $90^{\circ}$ then the similarity is 0 , and whether the vectors are diametrically opposite of each other then the similarity is -1 , independent of their magnitude [20]). Cosine similarity is specially used in non-negative space and the outcome lies within [0,1]. Given two vectors of attributes, $P$ and $Q$, the conise similarity, $\cos (\theta)$, is represented using a dot product and magnitude as

$$
\text { similarity }=\cos (\theta)=\frac{P \cdot Q}{\|P\|\|Q\|}=\frac{\sum_{i=1}^{n} P_{i} Q_{i}}{\sqrt{\sum_{i=1}^{n} P_{i}^{2}} \sqrt{\sum_{i=1}^{n} Q_{i}^{2}}}
$$

\section{B. Classification using Machine Learning}

In statistics, the classification being a supervised learning approach is one of the procedures of classifying a new set of records to which of a predefined class it belongs on the basis of a training data set with known category membership. Here the predefined classes can be identified by various methods such as machine learning.

Machine learning is a method used to build complex models that can give predictions where a training set of correctly defined observations is available [21].

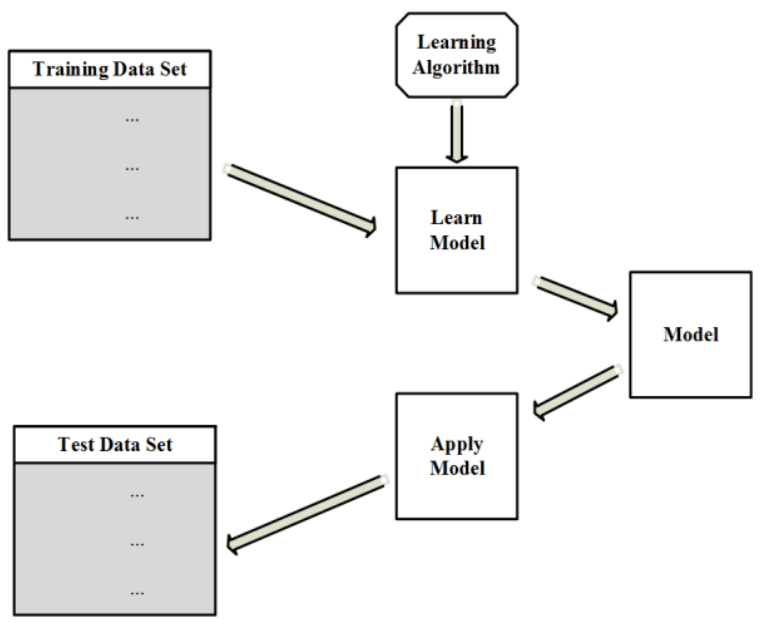

Fig.4. Classification using machine learning

These analytical models can help us to produce reliable inferences and hidden insights from the trends in the data [22].

\section{Lossless Compression}

Lossless compression is a type of data compression that allows the compressed data to be decompressed in a manner that will not have any data loss [23].

\section{1) DEFLATE Algorithm:}

Deflate [24] is an efficient lossless data compression algorithm that compresses data using a combination of the LZ77 [25, 26] algorithm and Huffman coding [27]. Several free and open source data compression applications (e.g. 7-Zip [28]) uses Deflate algorithm. The 
use of Deflate algorithm is also found in some popular file formats such as ZIP, gzip, PNG, PDF and so on.

\section{PROBLEM DESCRIPTION}

The communication between WSN and sensor gateway can be done by means of Bluetooth or wi-fi. On the other hand, the communication between cloud gateway and cloud environment can be of wired or wireless.

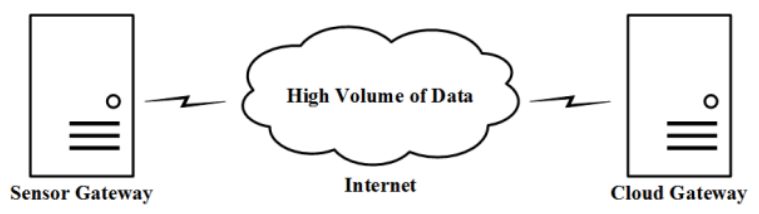

Fig.5. Target area of optimization

The most difficult part is the communication between sensor gateway and cloud gateway where a large-scale data is to be sent frequently. Since the internet is considered to be the communication medium, it requires a very high amount of bandwidth leading heavy transmission, high internet cost and lack of data security.

\section{PROPOSED FrAMEWORK}

As we know that sensor networks generally sense data in a periodic time interval, we can expect a lot of similarities in the same time period of the different cycle (a complete period of time) because the data generally follow seasonality.

In our proposed solution, instead of sending similar records (a set of sensor values in a specific time interval) again and again we prefer to send a single code which will represent the entire record and before sending it we are also using a compression algorithm (deflate algorithm [24]) which will reduce overall bandwidth requirement. The workflow of the proposed framework is depicted in Fig. 6.

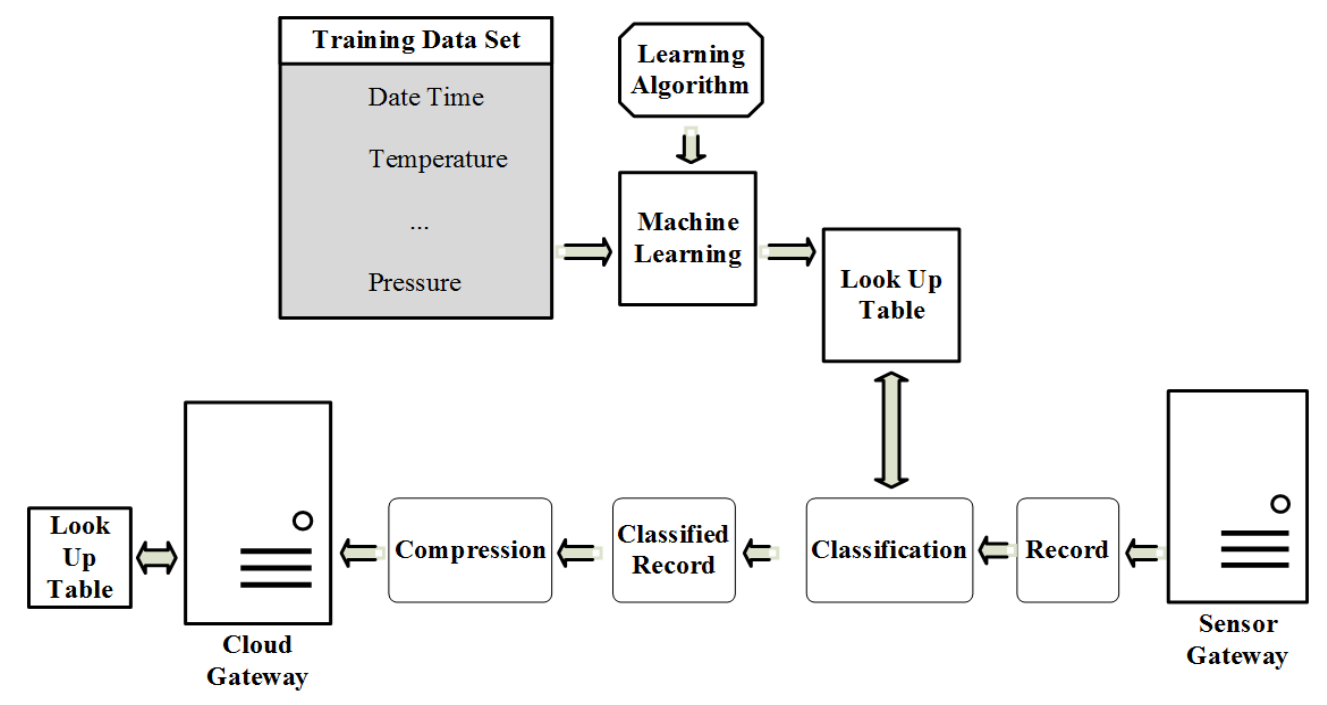

Fig.6. Flow diagram of proposed framework

First of all, we need a Look_Up_Table (a list of predefined records with individual codes) which will be used to measure the similarity. The Look_Up_Table can be built by mining (using machine learning approach) the prior data for a complete cycle.

At this point, the Look_Up_Table has to be available at both gateways (sending and receiving). For a new record, it should be compared with each record of the Look_Up_Table. If a standard amount of similarity is found, the corresponding code is sent instead of the total record. The dissimilar records will be added in the Look_Up_Table and after each cycle, the Look_Up_Table is to be updated based on the frequency of hit ratio.

\section{IMPLEMENTATION}

In our research, we have considered environmental data consisting of six sensors (Air Temperature, Dew
Point, Humidity, Pressure, Wind Speed, Sea Level Pressure) which are collected at one-minute interval [29]. We have obtained more than 5 lac records from the data source [29] for the one-year time frame.

\section{A. Time Series Analysis in order to find Seasonality}

As the data is collected at fixed time interval, we are considering it as a time series data which must have seasonal and cyclical effects. Here we are taking a single day as a cycle and each hour is considered as a season. As a result, there will be twenty-four seasons in a single cycle. For each month, we have calculated the seasonality to separate the overall seasonal effect of a year (complete cycle).

\section{1) Stationarity Test:}

First of all, we have identified the stationarity of the data using Dickey-Fuller (ADF) test.

Test interpretation: 
$H_{0}$ : There is a unit root.

$H_{a}$ : There is no unit root. The data is stationary.

Table 1. Stationary Test

\begin{tabular}{|c|c|}
\hline Parameter & Value \\
\hline Tau (Observed value) & -4.0525 \\
\hline Tau (Critical value) & -0.9079 \\
\hline p-value (one-tailed) & 0.0070 \\
\hline Alpha & 0.05 \\
\hline
\end{tabular}

As the computed p-value is lower than the significance level alpha $=0.05$, we should reject the null hypothesis $H_{0}$, and accept the alternative hypothesis $H_{a}$. The risk to reject the null hypothesis $H_{0}$ while it is true is lower than $0.70 \%$.

\section{2) Seasonality Measure:}

For a whole year we have considered each day as a cycle and each hour as a season, so we have measured the seasonal effect for each consecutive month as well as the complete cycle (a year).

We have calculated the seasonal indices for twentyfour seasons of every month which will explain the seasonal effect within them.

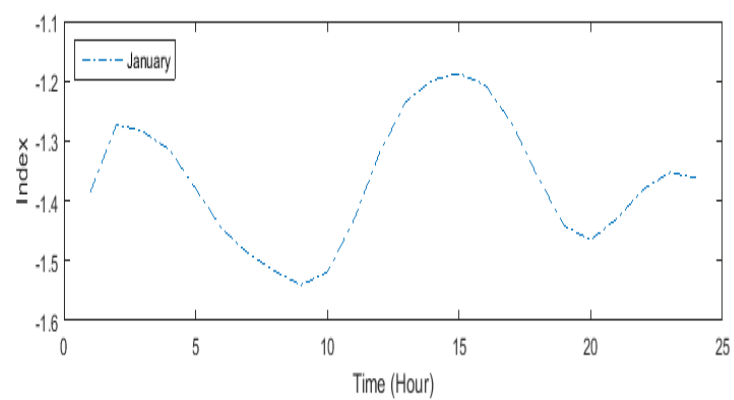

Fig.7. Seasonal index (January)

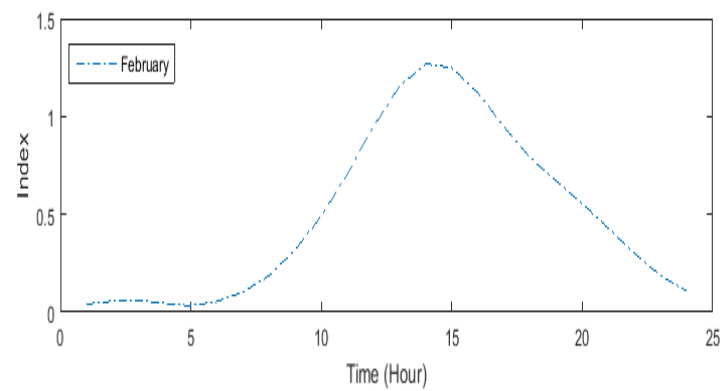

Fig.8. Seasonal index (February)

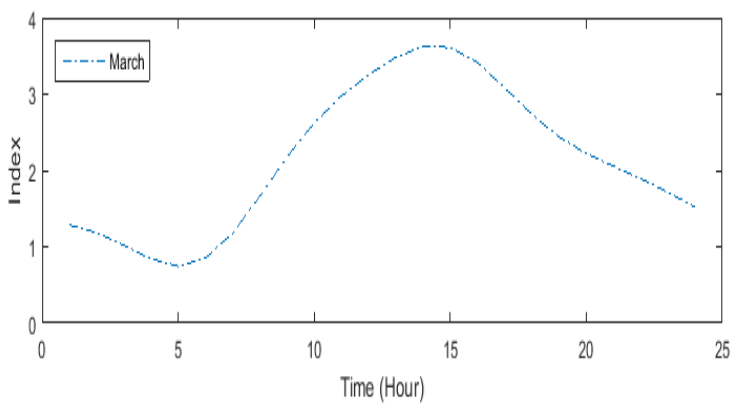

Fig.9. Seasonal index (March)

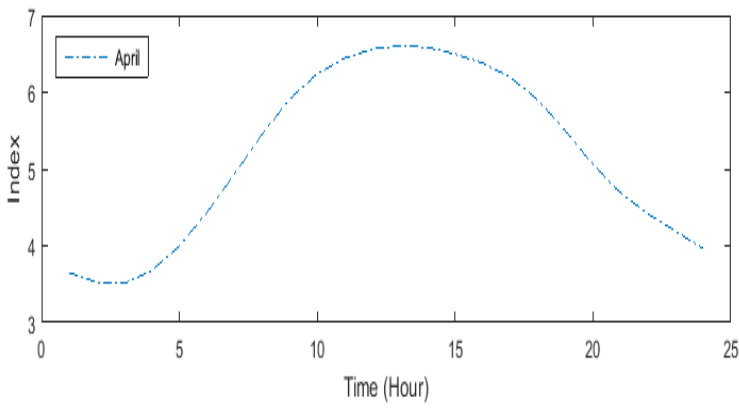

Fig.10. Seasonal index (April)

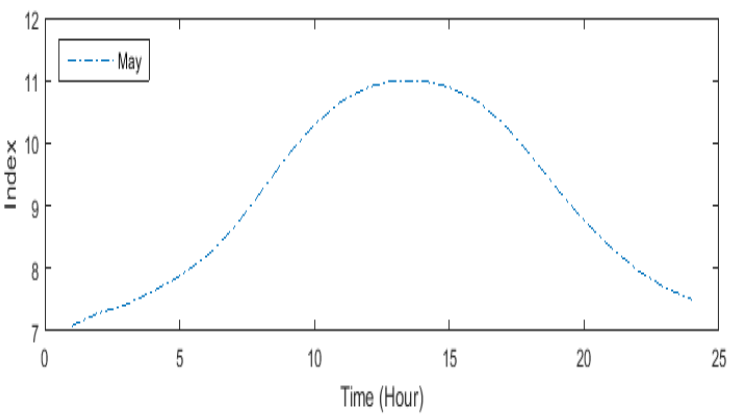

Fig.11. Seasonal index (May)

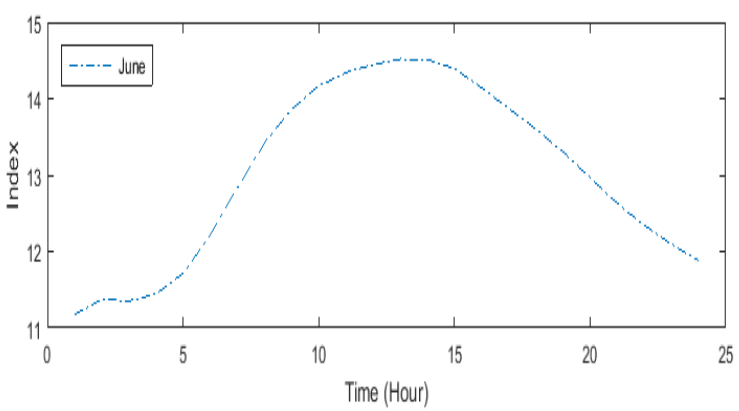

Fig.12. Seasonal index (June)

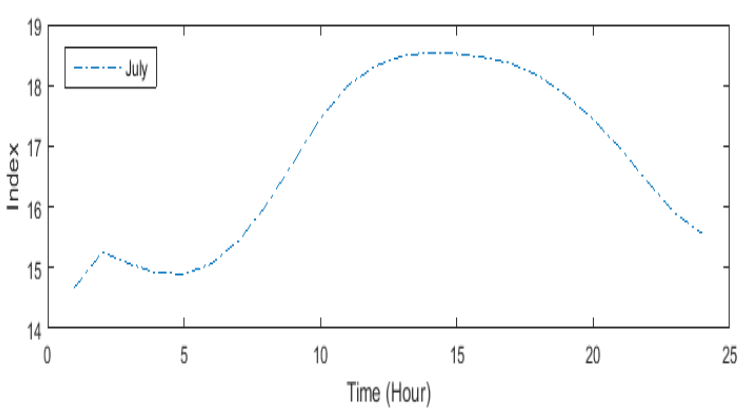

Fig.13. Seasonal index (July)

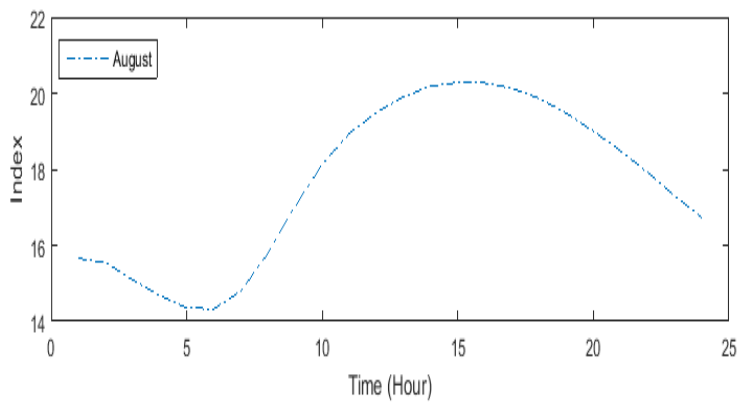

Fig.14. Seasonal index (August) 


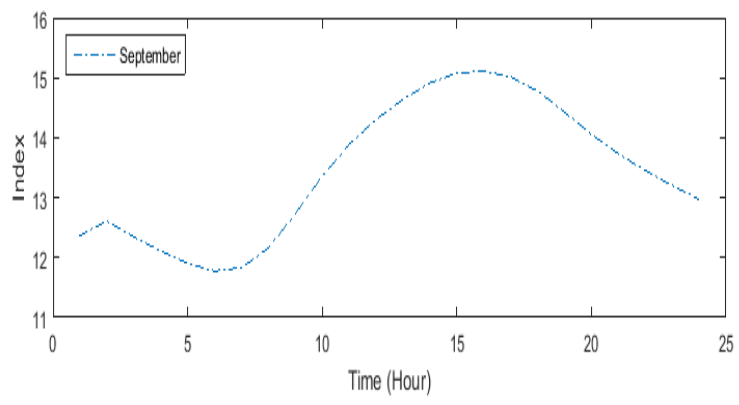

Fig.15. Seasonal index (September)

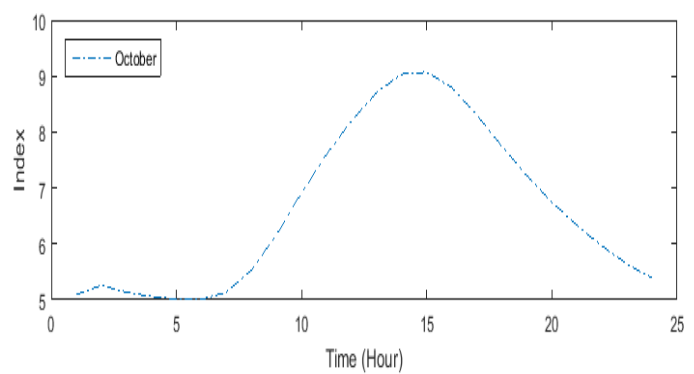

Fig.16. Seasonal index (October)

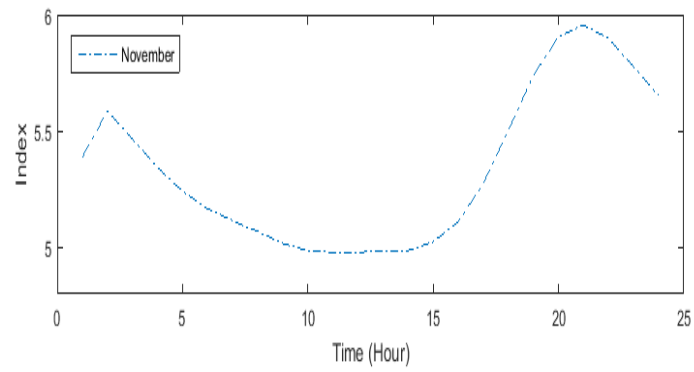

Fig.17. Seasonal index (November)

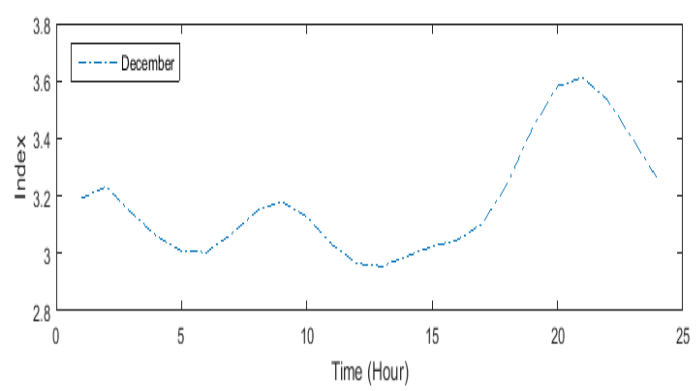

Fig.18. Seasonal index (December)

From Fig. 7-18, we can notice an existence of the almost similar pattern from February to October where the graph of seasonal indices follows a route from low to the zenith and resting at low again. For November to January, there exists another pattern having multiple ups and downs. Now we can conclude that there exists seasonality within successive months.

Since our main concern is to find similarity among each record in the data, so we have also identified the similarities between the successive months.

According to the fig. 19, we can see that there exist similar seasonal indices in between two halves of the year. As a result, we can expect that in spite of having similarity within each month there will also exist similarity among rest of them.

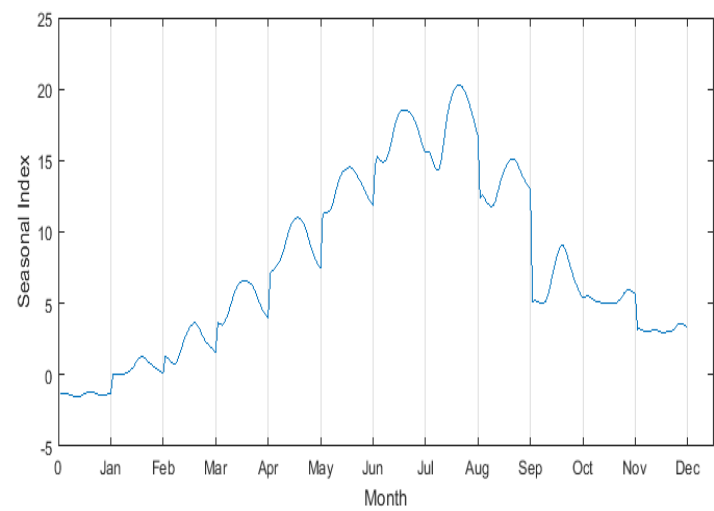

Fig.19. Overall seasonal index (Total Year)

\section{B. Generating the Look_Up_Table}

Since we have found seasonality at the previous step so it is obvious that there will be a lot of similarities in between successive seasons. For this reason, the Look_Up_Table has been generated using machine learning approach.

\section{Similarity Measure}

For each record, we have considered that to be a Look_Up_Table record if the Euclidean distance of it with every other record is less than a specific threshold, $\mathrm{k}$.

Here, $\mathrm{k}$ is the maximum allowed Euclidean distance which is determined by combining the standard deviations for all variables.

$$
\begin{gathered}
k=\frac{\sum_{i=1}^{m}\left(n_{i}-1\right) s_{i}^{2}}{\sum_{i=1}^{m}\left(n_{i}-1\right)} \\
k=\sqrt{\frac{\left(n_{1}-1\right) s_{1}^{2}+\left(n_{2}-1\right) s_{2}^{2}+\cdots+\left(n_{1}-1\right) s_{m}^{2}}{n_{1}+n_{2}+\cdots+n_{m}-m}}
\end{gathered}
$$

Using equation ( 3 ) we found $k \approx 4.6$ and we have got 1585 records in the Look_Up_Table which have classified about $98 \%$ of total data with a good hit ratio.

For each record, we have calculated the Euclidean distance with every other record. If the minimum distance is less than $k$ (threshold value) then we have considered the record as a new entry in the Look_Up_Table.

\section{Classification of new data}

At this step, we have got the Look_Up_Table for a complete cycle which will be used in order to classify new records. Now for the next cycle, when a new record appears, it has been compared with every Look_Up_Table records. If similarity (using previous similarity measure) is found, then only the corresponding code has been sent to cloud gateway and the frequency has been updated for that particular record. Otherwise, the raw record has been sent to the cloud gateway without any modification and considered as a new 
Look_Up_Table record.

After a complete cycle, we have an updated Look_Up_Table with different hit ratios. For the next complete cycle, we have eliminated the least used records from the table.

\section{RESULT AND DISCUSSION}

To measure the performance of the proposed framework, we have done the required simulations for different scenarios and the outcomes were also compared with the traditional approach and [5].

\section{A. Overall Bandwidth Comparison}

The primary concern of our research was to find the possibility of bandwidth reduction among sensor-cloud communication. For this reason, we have calculated the required bandwidth for every single transmission and found a significant difference between traditional and proposed approach. We have also compared the cumulative bandwidth for both cases (with and without compression) as well as with the traditional approach.

From fig. 20, we can see that a remarkable amount of bandwidth is being reduced by using our proposed framework.

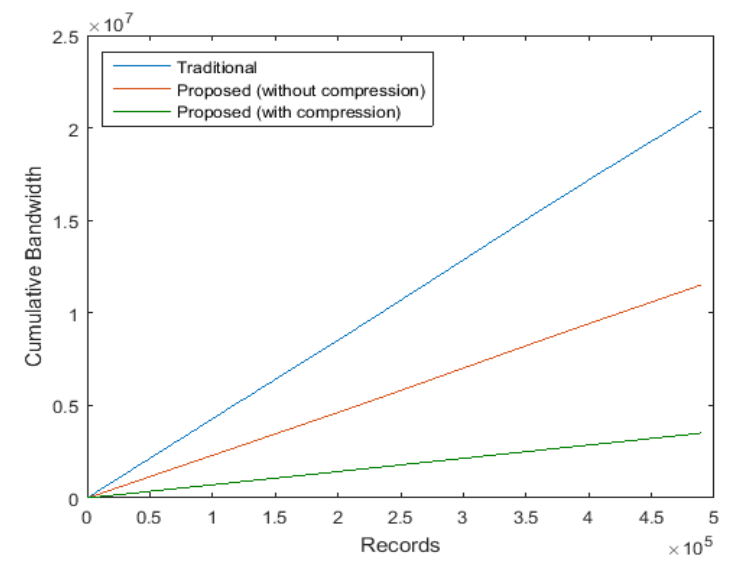

Fig.20. Cumulative Bandwidth Comparison

Though we have considered a limited number of sensors (i.e. six in our case), the result will be more noticeable when the network expands.

\section{B. Overall Time Comparison}

The time in which a transmission completes is very important in almost any type of communication. For this reason, a framework can be considered efficient if it reduces the bandwidth consumption and takes an acceptable amount of time for processing.

In our research, we have considered $128 \mathrm{Kbps}$ connection between the sensor and cloud gateway.

Here, $128 \mathrm{Kbps} \approx \frac{128}{8} k B p s$

$$
\begin{aligned}
& \approx 32 \mathrm{kBps} \\
& \approx 32 \times 1024 \text { bytes } / \mathrm{sec} \\
& \approx 32768 \text { bytes } / \mathrm{sec}
\end{aligned}
$$

According to equation 4 , we can say for any $x$ bytes we will need $x / 32768 \mathrm{sec}$ or $0.31 x$ millisecond. So it is clear that when the bandwidth increases the sending time also increases. For our proposed framework, we have measured the processing and sending time for every single record. The following graph describes the cumulative time comparison between the traditional and proposed approach.

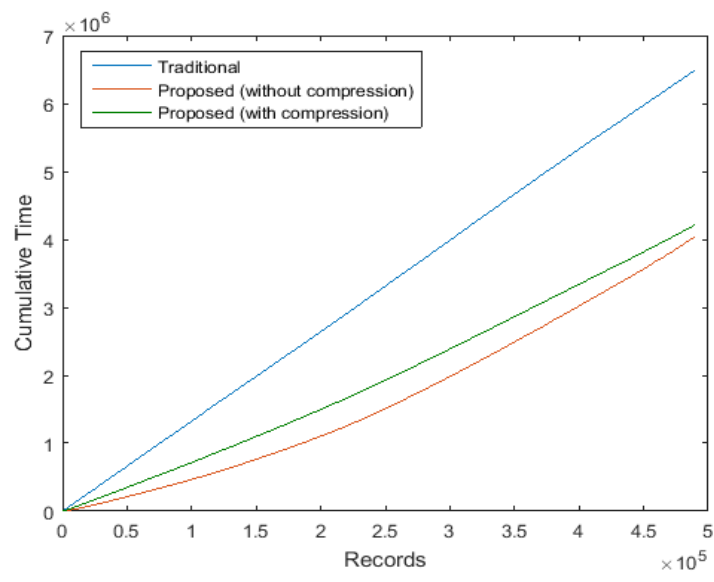

Fig.21. Cumulative Time (Processing and Sending) Comparison

According to fig. 21, we can see the time that our proposed framework (without compression) takes to process and send the data is less than the traditional approach. The graph also explains that the required time even after compression is less than the traditional approach and the difference between with and without compression is very minimal.

\section{Residual Measure}

After applying the framework, we have also compared the received data for both traditional and proposed approach in order to measure the changes.

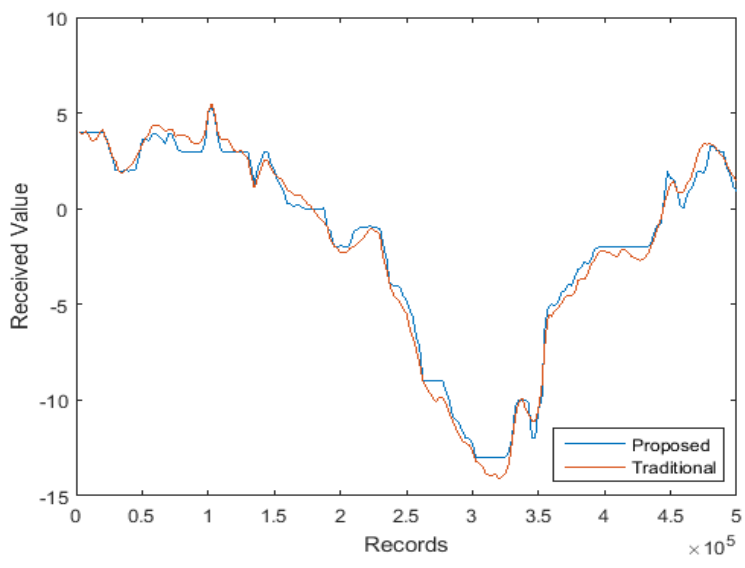

Fig.22. Residual Measure of Received Data

From fig. 22, we can see both lines are following the almost same path and the difference between them is negligible.

\section{Performance Comparison}

To compare with the result of [5], we have additionally 
calculated the required bandwidth for the first 15000 bytes using proposed framework with and without compression.

Table 2. Results of [5]

\begin{tabular}{|c|c|}
\hline Scenarios & Size in bytes \\
\hline Before compression & 15000 \\
\hline $\begin{array}{c}\text { After compression (without using } \\
\text { neural networks) }\end{array}$ & 980 \\
\hline $\begin{array}{c}\text { After compression (with using } \\
\text { neural networks) }\end{array}$ & 400 \\
\hline
\end{tabular}

Table 3. Results of Proposed Framework

\begin{tabular}{|c|c|}
\hline Scenarios & Size in bytes \\
\hline $\begin{array}{c}\text { Before classification and } \\
\text { compression }\end{array}$ & 15000 \\
\hline $\begin{array}{c}\text { After classification (without using } \\
\text { compression) }\end{array}$ & 1021 \\
\hline $\begin{array}{c}\text { After classification (with using } \\
\text { compression) }\end{array}$ & 85 \\
\hline
\end{tabular}

In our proposed framework, the bandwidth consumption without using compression is a bit higher than the framework proposed in [5]. But after using the compression algorithm, we have got a significant amount of bandwidth reduction and the data misclassification error is minor.

\section{CONCLUSION}

The combination of sensor-cloud is becoming very popular in recent years as it provides a new framework of accelerating service innovation and cross-disciplinary applications that span organizational boundaries. In sensor-cloud architecture, the communication between WSN and cloud computing is a challenging task as the required bandwidth is very high.

Our proposed framework can minimize the robust bandwidth requirement of sensor-cloud communication where the amount of data loss will be negligible and the required time for the transaction is also reduced to some extent. From our research, we have found that our proposed framework is providing 4.7 times better performance compared to related research.

We will continue to extend our research to observe the proposed framework in different real world application to optimize the overall performance.

\section{ACKNOWLEDGMENT}

We would like to express our gratitude to Heikki Junninen, Antti Lauri, Petri Keronen, Pasi Aalto, Veijo Hiltunen, Pertti Hari and Markku Kulmala for the data source although any errors are our own and should not denigrate the reputations of these persons.

\section{REFERENCES}

[1] W. Zeng, Y. Zhao, W. Song and K. Ou, "Research on Wireless Storage System Key Technologies", in 4th International Conference on Computer Sciences and
Convergence Information Technology, pp. 191-195, 2009.

[2] C. M. Sadler and M. Martonosi, "Data compression algorithms for energy-constraints devices in delay tolerant networks", in Proceedings of the 4th International Conference on Embedded networked sensor systems (SenSys '06), pp. 265-278, 2006.

[3] A. Scaglione and S. D. Servetto, "On the Interdependence of Routing and Data Compression in Multi-Hop Sensor Networks, "Wireless Networks, vol.11, no.1-2, pp. 149160, 2005.

[4] F. Marcelloni and M. Vecchio, "A Simple Algorithm for Data Compression in Wireless Sensor Networks," in IEEE Communications Letters, vol. 12, no. 6, pp. 411-413, 2008.

[5] L. P. Dinesh Kumar, S. Shakena Grace, A. Krishnan, V. M. Manikandan, R. Chinraj and M. R. Sumalatha, "Data filtering in wireless sensor networks using neural networks for storage in cloud," in Proceedings of the International Conference on Recent Trends In Information Technology (ICRTIT), pp. 202-205, 2012.

[6] A. Alamri, W. S. Ansari, M. M. Hassan, M. S. Hossain, A. Alelaiwi, and M. A. Hossain, "A Survey on Sensor-Cloud: Architecture, Applications, and Approaches," International Journal of Distributed Sensor Networks, vol. 2013, pp. 18, 2013.

[7] P. Kaur and V. Bhardwaj, "Wireless Sensor Networks: A Survey," International Journal of Advanced Research in Computer Science and Software Engineering, vol. 5, pp 988-994, 2015.

[8] Z. Jiang, X. Jin and Y. Zhang, "A Weather-Condition Prediction Algorithm for Solar-Powered Wireless Sensor Nodes," in Proceedings of the 6th International Conference on Wireless Communications Networking and Mobile Computing (WiCOM), pp. 1-4, 2010.

[9] G. Simon, M. Maroti, A. Ledeczi, G. Balogh, B. Kusy, A. Nadas, G. Pap, J. Sallai, K. Frampton, "Sensor networkbased counter sniper system," in Proceedings of the 2nd International Conference on Embedded Networked Sensor Systems (Sensys '04), pp. 1-12, 2004.

[10] J. Yick, B. Mukherjee and D. Ghosal, "Analysis of a prediction-based mobility adaptive tracking algorithm," in Proceedings of the 2nd International Conference on Broadband Networks (BROADNETS), vol. 1, pp. 753760, 2005.

[11] M. Castillo-Offer, D. H. Quintela, W. Moreno, R. Jordan and W. Westhoff, "Wireless sensor networks for flashflood alerting," in Proceedings of the Fifth IEEE International Caracas Conference on Devices, Circuits and Systems, pp. 142-146, 2004.

[12] T. Gao, D. Greenspan, M. Welsh, R. R. Juang and A. Alm, "Vital Signs Monitoring and Patient Tracking Over a Wireless Network," in 27th Annual International Conference of the Engineering in Medicine and Biology Society, pp. 102-105, 2005.

[13] K. Lorincz, D. J. Malan, T. R.F. Fulford-Jones, A. Nawoj, A. Clavel, V. Shnayder, G. Mainland, M. Welsh, S. Moulton, "Sensor Networks for Emergency Response: Challenges and Opportunities," IEEE Pervasive Computing, vol. 3, no. 4, pp. 16-23, 2004.

[14] S. K. Dash, J. P. Sahoo, S. Mohapatra, and S. P. Pati, "Sensor-cloud: assimilation of wireless sensor network and the cloud," in Advances in Computer Science and Information Technology. Networks and Communications, vol. 84, pp. 455-464, 2012.

[15] Harshi. K. Raj, "A Survey on Cloud Computing," International Journal of Advanced Research in Computer Science and Software Engineering, vol. 4, no. 7, pp. 352357, 2014. 
[16] J. Yick, B. Mukherjee, and D. Ghosal, "Wireless Sensor Network Survey," Computer Networks: The International Journal of Computer and Telecommunications Networking, vol. 52, no. 12, pp. 2292-2330, 2008.

[17] R. O. Duda , P. E. Hart , D. G. Stork, Pattern Classification (2nd Edition), Wiley-Interscience, 2000.

[18] E. Deza, M. M. Deza, Encyclopedia of Distances, Springer, pp. 94, 2009.

[19] P.-N. Tan, M. Steinbach and V. Kumar, Introduction to Data Mining, Addison-Wesley, ch. 8, pp. 500, 2005.

[20] I. S. Dhillon and D. S. Modha, "Concept Decompositions for Large Sparse Text Data Using Clustering". Machine Learning, vol. 42, no. 1-2, pp. 143-175, 2001.

[21] E. Alpaydin, Introduction to Machine Learning, MIT Press. p. 9, 2010.

[22] H. Mannila, "Data mining: machine learning, statistics, and databases," In Proceedings of the Eighth International Conference on Scientific and Statistical Database Management, pp. 2-9, 1996.

[23] D. Salomon, G. Motta, Handbook of Data Compression (5th edition), Springer, pp. 16-18, 2009.

[24] L. P. Deutsch, "Deflate compressed data format specification version 1.3," 1996.

[25] J. Ziv and A. Lempel, "A Universal Algorithm for Sequential Data Compression," in IEEE Transactions on Information Theory, vol. 23, no. 3, pp. 337-343, 1977.

[26] J. Ziv and A. Lempel, "Compression of individual sequences via variable-rate coding," in IEEE Transactions on Information Theory, vol. 24, no. 5, pp. 530-536, 1978.

[27] D. A. Huffman, "A method for the construction of minimum redundancy codes," in Proceedings of the IRE, vol. 40, no. 9, pp. 1098-1101, 1952.

[28] “7-Zip", [Online]. Available: http://www.7-zip.org/

[29] H. Junninen, A. Lauri, P. Keronen, P. Aalto, V. Hiltunen, P. Hari, M. Kulmala, "Smart-SMEAR: on-line data exploration and visualization tool for SMEAR stations", Boreal Environment Research (BER), vol. 14, pp. 447457, 2009.

\section{Authors' Profiles}

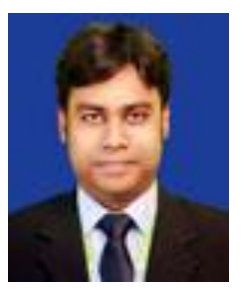

Md. T. Rahman has completed his B.Sc. (Hons.) in Information Technology from Institute of Information Technology, Jahangirnagar University, Savar, Dhaka, Bangladesh in 2013 and M.Sc. in Information Technology from the same institute in 2015. He is currently working as a Lecturer in the Department of Computer Science and Engineering, Daffodil International University from 2015. His research interest is in Wireless Communication, Cloud Computing, and Network Security.

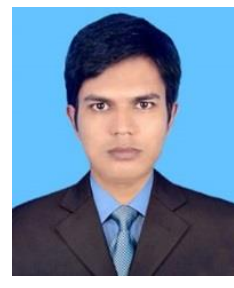

Md. Sifat A. Salan has completed his B.Sc. (Hons.) in Statistics from Department of Statistics, Jahangirnagar University, Savar, Dhaka, Bangladesh in 2014. He is currently conducting M.Sc. in Statistics in the same department. His research interest is in Multivariate Analysis, Time Series Analysis and Design of Experiment.

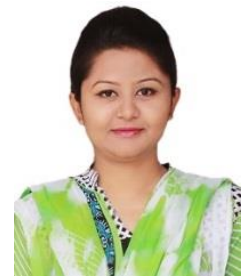

Taslima F. Shuva has completed her B.Sc. (Hons.) in Information Technology from Institute of Information Technology, Jahangirnagar University, Savar, Dhaka, Bangladesh in 2014. She is currently working as a Lecturer in the Department of Computer Science and Engineering, Daffodil International University from 2016. Her research interest is in Wireless Communication, Cloud Computing, and Network Security.

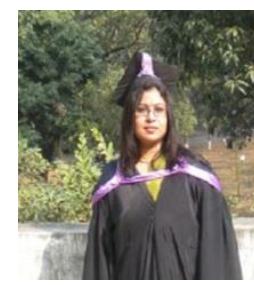

Risala T. Khan has completed her B.Sc. (Hons.) in Computer Science and Engineering from Jahangirnagar University, Savar, Dhaka in 2006 and M.Sc. in Computer Science and Engineering from the same University. She worked as a lecturer in the Department of Computer Science and Engineering, Daffodil International University, Dhaka since 2006 to 2009. She is now working as an Associate Professor at Institute of Information Technology, Jahangirnagar University, Savar, Dhaka, Bangladesh. Her research field is network traffic and network security.

How to cite this paper: Md. Tanvir Rahman, Md. Sifat Ar Salan, Taslima Ferdaus Shuva, Risala Tasin Khan,"Efficient Sensor-Cloud Communication using Data Classification and Compression", International Journal of Information Technology and Computer Science(IJITCS), Vol.9, No.6, pp.9-17, 2017. DOI: $10.5815 /$ ijitcs.2017.06.02 